\title{
Reactivity and mechanism of nucleophilic addition reaction of amine with alkene: A systematic DFT study
}

\author{
Zouhair Lakbaibi ${ }^{1, *}$, Adil Jaafar ${ }^{2}$, Hicham Ben El Ayouchia ${ }^{3, *}$, Mohamed Tabyaoui ${ }^{1}$ and \\ Abdelghani Boussaoud ${ }^{2}$ \\ ${ }^{\mathbf{1}}$ Laboratory of Materials, Nanoparticles and Environment, University of Mohammed V Faculty of Sciences, \\ Department of Chemistry, BP 1014 Avenue Ibn Batouta Rabat Morocco \\ ${ }^{2}$ Laboratory of Process, Signals, Industrial Systems and Computer Science, Superior School of Technology, \\ Cadi Ayyad University, Dar Si-Aïssa road, 46000, BP 89, Safi, Morocco \\ ${ }^{3}$ Laboratoire de Chimie Analytique et Moléculaire, - LCAM, Faculté Polydisciplinaire de Safi, Université Cadi \\ Ayyad, Safi 46030, Morocco
}

\begin{abstract}
The reactivity and mechanism of the nucleophilic addition reaction of diethylamine 1 and 1-cyano-2phenyl vinyl methane sulfonate $\mathbf{2}$ have been studied for a systematic understanding of this relevant organic transformation, using DFT calculations method at the B3LYP/6-311G(d,p) computational levels. Analysis of the conceptual DFT reactivity indices allows explaining the reactivity, and the calculated nucleophilic and electrophilic Parr functions at the reactive sites of reagents $\mathbf{1}$ and $\mathbf{2}$, respectively, allows explaining correctly the regioselectivity observed experimentally. The study has also been applied to predict the mechanism of the amine with an alkene. Interestingly, the study predicts a switch to a two-step mechanism due to the higher polar character of this zw-type nucleophilic addition reaction.
\end{abstract}

Keywords: Nucleophilic Addition reaction; Parr Function; Reactivity; mechanism; amine; alkene; DFT; nucleophilivity/electrophilicity.

\section{Introduction}

Nucleophilic addition of $\mathrm{N}-\mathrm{H}$ bond of an amine to carbon-carbon multiple bonds of an alkene, alkyne, diene, or allene ${ }^{1,3}$, in the case of the reaction between alkene and amine occurs in two steps. The first step involves the nucleophilic addition of the amine compound on the carbon double bonds of an alkene, which results in the zwitterionic intermediate. The second one is intramolecular proton transfer from amine moiety to negatively charged carbon leads to corresponding product ${ }^{1}$. Usually, the addition of amines to ethylenic compounds has been performed under the solvating system (dichloroethane or acetonitrile) ${ }^{4}$.

In particular, the nucleophilic addition of diethylamine on the 1-cyano-2-phenyl vinyl methanesulfonate $\mathbf{2}$ could lead to a product $\mathbf{P}$ named 1-cyano-2-diethylamino-2-phenylethyl

methanesulfonate (Scheme 1), this product presents three functional groups such as methanesulfonate, cyano and amine which are considered in the organic chemistry literature as a versatile route for the synthesis of an excellent intermediates to synthesize numerous products with a wide range of biological activity ${ }^{5,7}$. Our main goal in this work is to give a

*Corresponding author: Zouhair Lakbaibi / Hicham Ben El Ayouchia

Email address: zouhairshaim@gmail.com / belayou@gmail.com

DOI: http://dx.doi.org/10.13171/mjc811902924zlhbea theoretical study of the reaction between 1-cyano-2phenyl vinyl methanesulfonate (2) and diethylamine (1) using density functional theory (DFT) ${ }^{8}$, and this allows to explain quantitatively some chemical and physical properties related to this reaction, such as chemical reactivity, chemical kinetics, global electron density transfer (GEDT) in the transition state, etc. Then, the knowledge of its properties is necessary for an experimental chemist in the prediction of the reaction feasibility. In this context, a computational study has become necessary to compare the theoretical results with those obtained experimentally and sometimes to complete experimental measurements or even to supplement them when they cannot be realized.

\section{Computational methods}

All stationary points involved in the nucleophilic addition reaction were optimized using the B3LYP functional ${ }^{9,10}$ together with the standard 6-311G(d,p) basis set ${ }^{11}$. Frequency computations characterized the stationary points in order to verify that TSs have only one imaginary frequency ${ }^{12}$.

Acetonitrile solvent was implicitly modelled 
using the conductor-like polarizable continuum model (CPCM) developed by Tomasi ${ }^{13,14}$ in the framework of the self-consistent reaction field (SCRF) ${ }^{15,16}$. The global electron density transfer (GEDT) ${ }^{17}$ at the TSs was computed by the sum of the natural atomic charges, obtained through a natural population analysis (NPA) ${ }^{18}$ of the atoms belonging to either the nucleophilic or electrophilic framework. All computations were carried out with the Gaussian 09 suite of programs ${ }^{19}$.

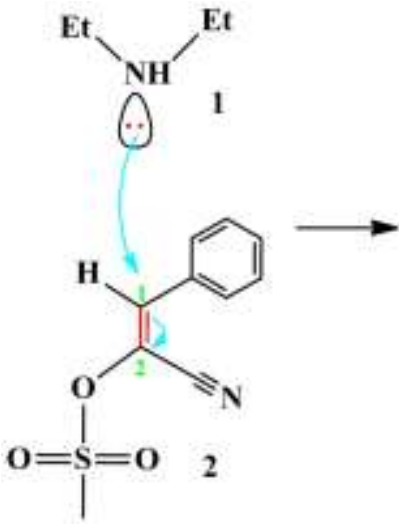

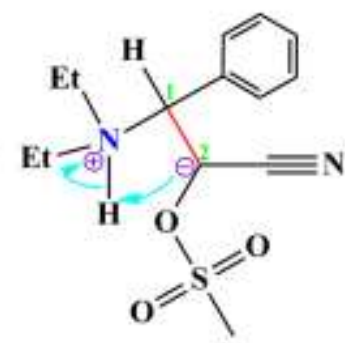

IN

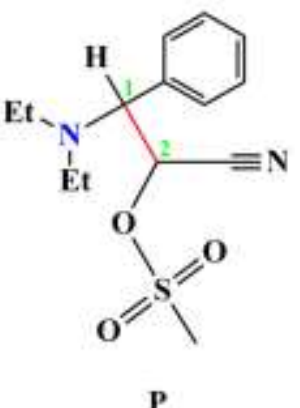

$\mathbf{P}$

Scheme 1. Nucleophilic addition of diethylamine on the 1-cyano-2-phenyl vinyl methanesulfonate.

The global electrophilicity index, $\omega$, is given by the following expression, $\omega=(\mu 2 / 2 \eta)^{20}$, in terms of the electronic chemical potential, $\mu$, and the chemical hardness, $\eta$. Both quantities may be approached in terms of the one-electron energies of the frontier molecular orbitals HOMO and LUMO, $\varepsilon_{\mathrm{H}}$ and $\varepsilon_{\mathrm{L}}$, as $\mu \approx\left(\varepsilon_{\mathrm{H}}+\varepsilon_{\mathrm{L}}\right) / 2$ and $\eta=\left(\varepsilon_{\mathrm{L}}-\varepsilon_{\mathrm{H}}\right)$, respectively ${ }^{21,22}$. The empirical (relative) nucleophilicity index $N$, based on the HOMO energies obtained within the Kohn-Sham scheme is defined as $N=\varepsilon_{\mathrm{HOMO}}(\mathrm{Nu})-\varepsilon_{\text {Hомо }}(\mathrm{TCE})$ ${ }^{21,22}$, where tetracyanoethylene (TCE) is the reference because it presents the lowest HOMO energy in a long series of molecules already investigated in the context of polar organic reactions. Nucleophilic $P_{k}^{-}$and electrophilic $P_{k}^{+}$Parr functions were obtained through the analysis of the Mulliken atomic spin density (ASD) of the radical cation and anion, respectively ${ }^{23}$.

\section{Results and Discussion}

\section{Global and local reactivity analysis of the reagents}

Numerous theoretical studies have shown that the analysis of the reactivity indices defined within the conceptual DFT (CDFT) ${ }^{24}$ is a powerful method to understand the reactivity in polar reactions. Consequently, the global CDFT indices, namely, the electronic chemical potential, $\mu$, chemical hardness, $\eta$, electrophilicity, $\omega$, and nucleophilicity, $N$, of diethylamine $\mathbf{1}$ and the 1-cyano-2-phenyl vinyl methanesulfonate $\mathbf{2}$ are given in Table 1 .

Table 1. B3LYP/6-311G(d,p) electronic chemical potential $\mu$, chemical hardness $\eta$, global electrophilicity $\omega$ and global nucleophilicity $N$, in $\mathrm{eV}$, of amine $\mathbf{1}$ and alkene $\mathbf{2}$.

\begin{tabular}{|c|c|c|c|c|}
\hline & $\boldsymbol{\mu}$ & $\boldsymbol{\eta}$ & $\boldsymbol{\omega}$ & $\boldsymbol{N}$ \\
\hline $\mathbf{1}$ & -3.74 & 5.03 & 1.39 & 2.18 \\
\hline $\mathbf{2}$ & -4.61 & 4.46 & 2.38 & 2.72 \\
\hline
\end{tabular}

The electronic chemical potential of $\mathbf{1}, \mu=-3.74$ $\mathrm{eV}$, is higher than that of $2, \mu=-4.61$, indicating that along with a polar reaction the global electron density transfer (GEDT) will take place from the diethylamine $\mathbf{1}$ to the 1-cyano-2-phenyl vinyl methanesulfonate $\mathbf{2}$. In good agreement with the GEDT computed at the TSs (see later). The diethylamine $\mathbf{1}$ is classified as a moderate electrophile $\omega=1.39 \mathrm{eV}$, and a moderate nucleophile, $N=2.18 \mathrm{eV}$ within the $\omega$ and $N$ scale $^{25}$. The 1-cyano-2-phenyl vinyl methanesulfonate $\mathbf{2}$ has an electrophilicity $\omega$ index of $2.38 \mathrm{eV}$, is classified as a strong electrophile, and a nucleophilicity index of $2.72 \mathrm{eV}$, is classified as a moderate nucleophile ${ }^{25}$.
The high electrophilic character of 1-cyano-2phenyl vinyl methanesulfonate $\mathbf{2}$ and the high nucleophilic character of diethylamine $\mathbf{1}$ make that alkene will act as the electrophile and amine as the nucleophile in this reaction with a large polar character.

Along with a polar reaction involving the participation of non-symmetric reagents, the most favourable reactive channel is that involving the initial two-center interaction between the most electrophilic center of the electrophile and the most nucleophilic center of the nucleophile. Recently, Domingo proposed the electrophilic $P_{k}^{+}$and 
nucleophilic $P_{k}^{-}$Parr functions as powerful tools in the study of the local reactivity in polar processes. Hence, in order to characterise the most nucleophilic and the most electrophilic centers of the species involved in this nucleophilic addition reaction, and, thus, to explain the regioselectivity experimentally

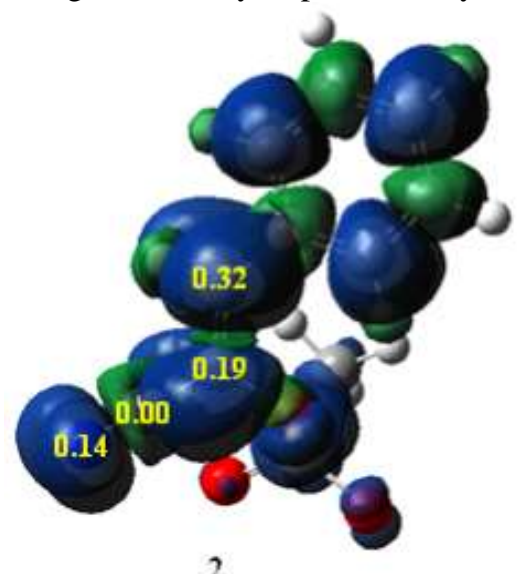

observed, the electrophilic $P_{k}^{+}$Parr functions of 1-cyano-2-phenyl vinyl methanesulfonate 2 , and the nucleophilic $P_{k}^{-}$Parr functions of diethylamine $\mathbf{1}$ were analysed (Figure 1).

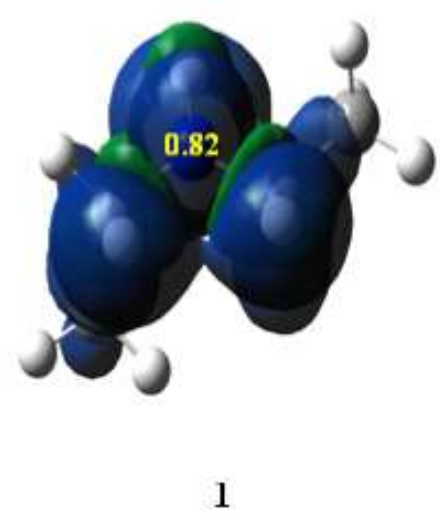

Figure 1. 3D representation of the Mulliken ASD of radical cations of pyrazoles and the radical anion of an alkene, including the nucleophilic $P_{k}^{-}$Parr functions of amine 1, and the electrophilic $P_{k}^{+}$Parr functions of alkene 2 .

Analysis of the nucleophilic $P_{k}^{-}$Parr functions of diethylamine 1 indicates that the amine N1 nitrogen, $P_{k}^{+}=0.82\left(N_{\mathrm{N} 1}=1.78 \mathrm{eV}\right)$ is the most nucleophilic center of this species. On the other hand, analysis of the electrophilic $P_{k}^{+}$Parr functions of 1-cyano2-phenyl vinyl methanesulfonate 2 indicates that the $\mathrm{C} 1$ carbon, $P_{k}^{+}=0.32\left(\omega_{\mathrm{C} 1}=0.76 \mathrm{eV}\right)$ is also the most electrophilic center. Consequently, the most favourable nucleophilic/electrophilic two centres interaction along $\mathrm{C}-\mathrm{N}$ single bond formation will take place between the $\mathrm{N}$ nitrogen atom of amine and the $\mathrm{C} 1$ carbon atom of the alkene.

Mechanistic study of the nucleophilic addition reaction of diethylamine 1 with 1-cyano-2-phenyl vinyl methanesulfonate 2

Analysis of the stationary points involved in the reaction between 1-cyano-2-phenyl vinyl methanesulfonate and diethylamine in acetonitrile as solvent indicates that this nucleophilic addition reaction takes place through a stepwise mechanism, two TSs, TS1 and TS2, and one intermediate, IN, have been located and characterized (Figure 2). In the first step of this mechanism is a nucleophilic attack to the carbon atom of alkene via the nitrogen atom of amine, forming an intermediate, IN. The calculated barrier is $10.67 \mathrm{kcal} \mathrm{mol}^{-1}$. Whereas the formation of the zwitterionic intermediate $\mathbf{I N}$ is located $1.06 \mathrm{kcal} / \mathrm{mol}$ below than TS1. In the second step, the $\mathrm{C} 2$ carbon of the alkene in IN rips off the ammonium proton, forming the corresponding product. The calculated barrier is $38.66 \mathrm{kcal} \mathrm{mol}^{-1}$. This step corresponds to the rate-determining step of the stepwise process.

The geometries of the TSs associated with the nucleophilic addition reaction between 1-cyano-2phenyl vinyl methanesulfonate and diethylamine are given in Figure 3 . The lengths of the $\mathrm{C}-\mathrm{N}$ forming bonds at the TSs are: $1.708(\mathrm{C} 1-\mathrm{N}) \AA$ at $\mathrm{TS} 1$ and $1.551(\mathrm{C} 1-\mathrm{N}), 1.313(\mathrm{~N}-\mathrm{H})$ and $1.474(\mathrm{C} 2-\mathrm{H}) \AA$ at TS2.

Numerous studies have shown a strong relationship between the polar character and the feasibility of organic reactions; the larger the GEDT at the TS is, the more polar and thus, faster, the reaction. In order to evaluate the electronic nature, i.e. polar or non-polar of the nucleophilic addition reaction between 1-cyano-2-phenyl vinyl methanesulfonate and diethylamine, the GEDT at the TSs was analysed. The resulting values are reported in Figure 3. The natural charges at the TSs appear to be shared between the 1-cyano-2-phenyl vinyl methanesulfonate and diethylamine. The GEDT, which fluxes from amine to alkene at the TSs, is $0.45 \mathrm{e}$ at TS1 and $0.43 \mathrm{e}$ at TS2. These very high values indicate that these TSs have a polar character, in agreement with the high electrophilic character of 1-cyano-2-phenyl vinyl methanesulfonate and the high nucleophilic character of diethylamine. 


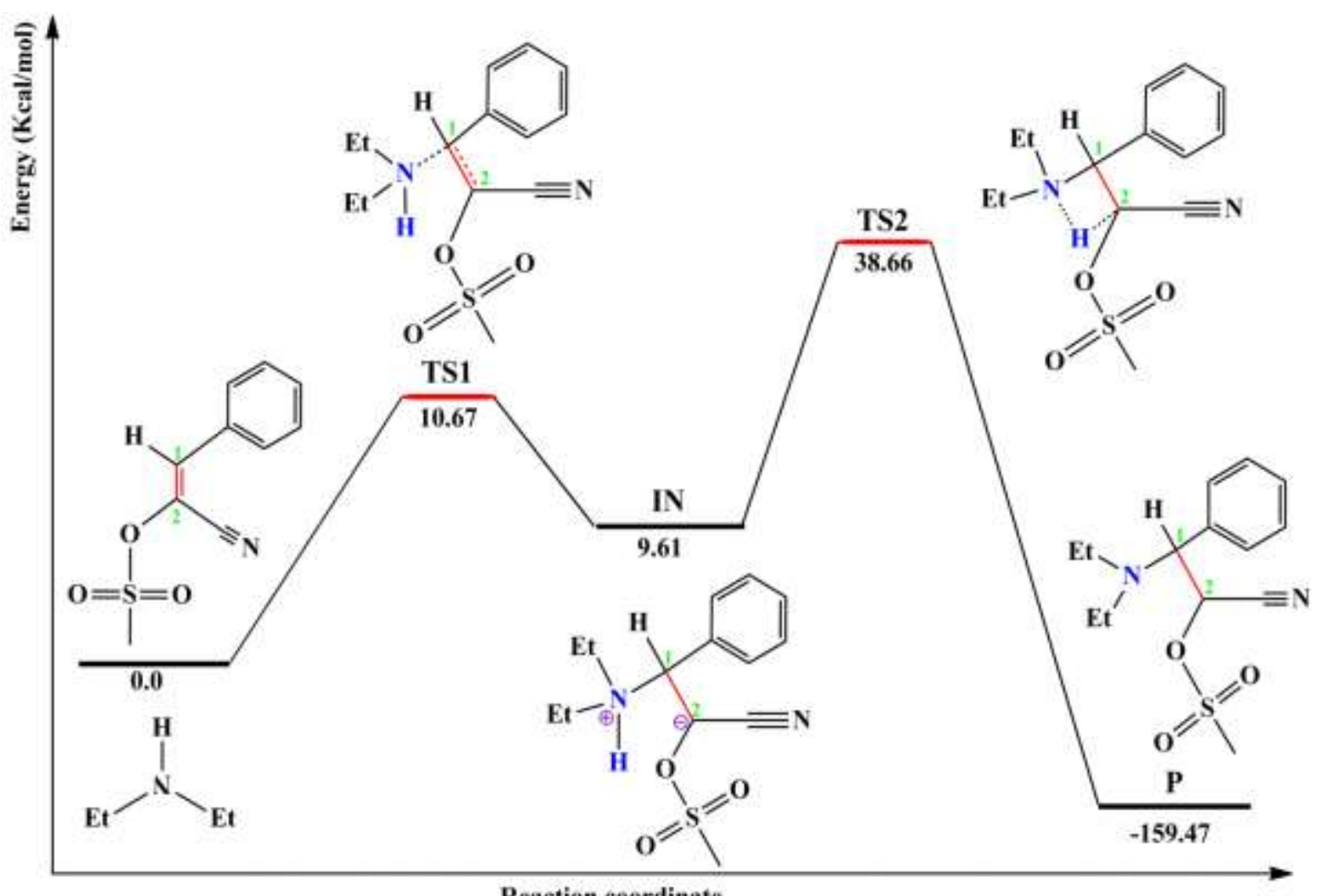

Reaction coordinate

Figure 2. Schematic representation (energy vs reaction coordinate) of the nucleophilic addition reaction between 1-cyano-2-phenyl vinyl methanesulfonate and diethylamine in acetonitrile.

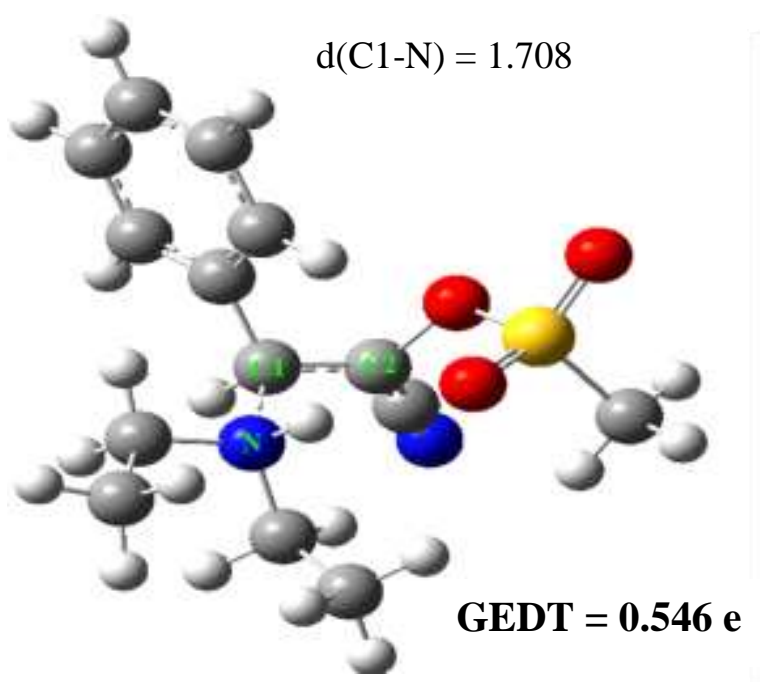

TS1

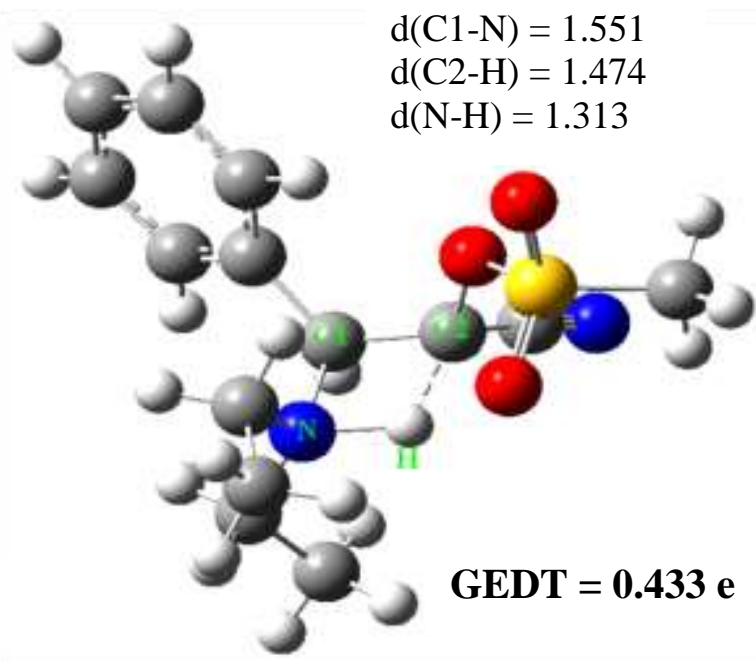

TS2

Figure 3. Transition states of the reaction between 1-cyano-2-phenyl vinyl methanesulfonate and diethylamine. The lengths are given angstroms.

\section{Conclusion}

The reactivity and regioselectivity of the nucleophilic addition reactions of 1-cyano-2-phenyl vinyl methanesulfonate with diethylamine leading to 1-cyano-2-diethylamino-2-phenylethyl methane sulfonate have been theoretically studied within the DFT calculations at the B3LYP/6-311 G(d,p) computational level.
Analysis of the CDFT reactivity indices of the amine and alkene allows characterizing the electrophilic/nucleophilic behaviours of these species. Analysis of the nucleophilic $P_{k}^{-}$and electrophilic $P_{k}^{+}$Parr functions indicates that the most favourable nucleophilic/electrophilic two-center interaction along an asynchronous $\mathrm{C} 1-\mathrm{N}$ 
single bond formation will take place between the nitrogen atom of the amine and the $\mathrm{C} 1$ carbon of the alkene. Formation of the 1-cyano-2-diethylamino-2phenyl ethyl methane sulfonate present high activation energies and takes place through a zwitterionic mechanism with formation of an intermediate.

\section{References}

1- T. E. Müller, M. Beller, Chemical Reviews, 1998, 98, 675-704.

2- Kai C. Hultzsch, Organic and Biomolecular Chemistry, 2005, 3, 1819-1824.

3- T. E. Muller, K. C, Hultzsch, M. Yus, F. Foubelo, M. Tada, Chem. Rev, 2008, 108, 3795-3892.

4- C. R. Brindaban, S. D. Suvendu, H. Alakananda, 2002, 5, 76-81.

5- G. R. Westerhof, R. E. Ploemacher, A. Boudewijn, I. Blokland, J. H. Dillingh, A.T. Mc Gown, J. A. Hadfield, M. J. Dawson, J. D. Down, CANCER RESEARCH, 2000, 60, 5470-5478.

6- Lin. Tai-Shun, H. Prusoff. William, J. Med. Chem, 1978, 21, 109-112.

7- F. Fraser. Fleming, Lihua Yao, P.C. Ravikumar, Lee Funk, C. Shook. Brian, J Med Chem, 2010, 53, 7902- 7917.

8- P. Geerlings, F. De Proft, W. Langenaeker, Chem. Rev, 2003, 103, 1793-1874.

9- A. D. Becke, J. Chem. Phys, 1993, 98, 5648-5652.

10- C. Lee, W. Yang, R. G. Parr, Phys Rev B, 1988, 37, 785-789.
11- W. J. Hehre, L. Radom, P. Schleyer, J. A. Pople, J. Comput. Chem, 1986, 7, 379-383.

12- C. Gonzales, H. B. Schlegel, J. Chem. Phys, 1989, 90, 2154-2161.

13- J. Tomasi, M. Persico, Chem Rev, 1994, 94, 2027-2094.

14- B. Y. Simkin, I. Sheikhet, Ellis Horwood, London, 1995.

15- E. Cances, B. Mennucci, J. Tomasi. J Chem Phys, 1997, 107, 3032-3041.

16- V. Barone, M. Cossi, J. Tomasi. J Comput Chem, 1998, 19, 404-417.

17- L. R. Domingo, RSC Adv, 2014, 4, 32415-32428.

18- A. Benallou, Z. Lakbaibi, H. Garmes, H. El Alaoui EL Abdallaoui, J. Fluorine. Chem., 2019, 219, 79-91.

19- M. J. Frisch et al., GAUSSIAN 09, Revision E. 01, Gaussian Inc., Wallingford CT, 2009.

20- P. Geerlings, F. De Proft, W. Langenaeker, Chem. Rev, 2003, 103, 1793-1874.

21- Z. Lakbaibi, H. Abou EL Makarim, M. Tabyaoui, A. EL Hajbi, Journal of Materials and Environmental Science, 2016, 8, 99-115.

22- Z. Lakbaibi, H. Abou EL Makarim, M. Tabyaoui, A. EL Hajbi, Moroccan Journal of Chemistry, 2016, 4, 437-453.

23- L. R. Domingo, M. R. Gutérrez, P. Pérez, J. Org. Chem, 2018, 83, 2182-2197.

24- L. R. Domingo, M. J. Aurell, P. Pérez, R. Contreras, Tetrahedron, 2002, 58, 4417-4423.

25- L. R. Domingo, M. R. Gutiérrez, B. Silvi, P. Pérez, Chem. Eur. J, 2018, 9, 1107-1120. 